DOI: $10.17805 /$ zpu.2015.4.16

\title{
Становление психологической темпометрии как нового научного направления в психологии
}

\author{
С. В. ТИХОМИРОвА \\ (ИнстИтУт пСИХологиИ РАН)
}

Проблема субъективного времени в психологии разрабатывается уже длительное время. В последние 50 лет наблюдается решительный рост количества исследований субъективного времени в психологии. Современную психологию, как отечественную, так и зарубежную, больше интересует психофизиологическая сторона процесса восприятия времени в норме и патологии, нежели онтогенетическая сторона. В основном исследования касаются роли различных психических функций в процессе восприятия времени: внимания, памяти и мышления, а также церебральных структур. В статье изложены основы нового направления в психологии - темпометрии. Естественнонаучный подход к изучению субъективного времени впервые применяется в отечественной психологии.

Качественно новым решением является переход от изучения восприятия времени к собственно изучению феномена субъективного времени. Взаимосвязь и взаимовлияние процессов восприятия времени и выбор между субъективными временами также являются предметом исследований в психологической темпометрии.

Теоретические основания психологической темпометрии связаны с работами С. Л. Рубинштейна. Несмотря на то что С. Л. Рубинштейн в целом оставался в парадигме исследования феномена восприятия субъективного времени, автор статьи считает необходимым отметить важность всех теоретических подсказок, данных С. Л. Рубинштейном в главе «Восприятие времени», являющейся не только главой лучшего учебника психологии «Основы общей психологии», но и глубоким анализом проблемы психологического времени. Важнейшими теоретическими положениями С. Л. Рубинштейна, которые будут развиты в психологической темпометрии, являются понятие вектора применительно к субъективным временам, проблема «текучей диалектики временных определений», понятие напряжения временного отрезка, закон заполненных временных отрезков, координатная система единиц измерений субъективного времени, привязанная к естественной отправной точке, коей является настоящее мгновение; приоритетная установка на будущее в переживаемом в настоящем мгновении времени.

Ключевые слова: темпометрия; субъективное время; естественнонаучный подход; С. Л. Рубинштейн; восприятие времени

\section{BВЕАЕНИЕ}

$\mathrm{B}$ заимопонимание людей основано на объективности, на общей картине мира и на использовании единых мер в пространственно-временных измерениях. Сообществу людей нужны единые меры, нивелирующие индивидуальные человеческие различия в измерениях, нужны эталоны, с которыми можно соотносить скорости, темпы и ритмы собственной деятельности и жизни. Конвенциальное время - это общественный договор, соглашение, а именно общий эталон, о котором человеческое сообщество договаривалось по-разному в разные времена. С 1967 г. в качестве эталона времени используется секунда, которая равна 9192631770 периодам излучения, соответствующего переходу между двумя сверхтонкими уровнями основного состояния атома цезия-133. В любом случае, договорное время всегда было связано с объективными физическими процессами.

Наличие общественно-согласованного эталона времени обеспечивает возможность технического прогресса, феноменальные успехи физических наук, достижения

* Публикация подготовлена при поддержке РФФИ, грант 15-06-08249.

This publication has been produced with the support of the Russian Foundation for Basic Research, grant 15-06-08249. 
в освоении космоса и исследованиях структуры материи. Без социального стандарта, согласованного эталона объективного времени развитие человечества шло бы значительно медленнее.

Эта договорная условность выросла из необходимости сравнения разных субъективных времен и трудностей взаимопонимания, вытекающих из разного течения субъективных времен у различных людей. Причем согласованный социумом эталон времени основан не на человеческих циклах и ритмах, а привязан к ритмическим процессам движения небесных тел и колебаний неживой материи, т. е. их объективной физической реальности, в которой человек живет.

Таким образом, в данной статье предлагается новый ракурс, новый подход к изучению субъективного времени, в котором внимание обращено на субъективные времена, ориентацию субъекта в векторах своих субъективных времен и на продуцирование временных интервалов самим субъектом и их изучение в разных отношениях, связях, обстоятельствах, в теоретических и эмпирических исследованиях через включение в новые связи и отношения.

\section{ПРОБАЕМЫ ИЗУЧЕНИЯ СУБЪЕКТИВНОГО ВРЕМЕНИ В ПСИХОАОГИИ}

Психология изучает субъективное время длительно в различных отраслях. В основном исследуются вопросы восприятия времени, т. е. восприятие объективной физической реальности. Также время рассматривается в психологических исследованиях процессуальных характеристик социально-психологических и психологических феноменов. Время в психологии чаще выступает как внешний фактор, средство, условие исследования различных феноменов психики. Исключительно редко время бывает не фактором, условием, мерилом, а полноценным объектом изучения. Например, в психологии разрабатывается ресурсный подход к изучению времени (АбульхановаСлавская, 1991; Зинченко, 2001). Время определяется как один из важнейших человеческих ресурсов и ограничений, а также одно из основных измерений пространства, в котором живет человек. «Человек всегда находится в живом, жизненном времени, пишет В. П. Зинченко, - которое отличается от хронологического времени жизни. Жизненное время определяет и жизненное пространство, жизненный мир человека» (Зинченко, 2001: 36). Развитие закона заполненного временного отрезка творчески развито и продолжено ученицей С. $\Lambda$. Рубинштейна К. А. Абульхановой, которая в личностное временя включила и идеальное, ценностное время-пространство, что позволило рассмотреть проблему развития личности в жизненном пути: «Ценностное отношение к жизни проявляется в мотиве успеть воплотить себя в жизни, в чем-то непреходящем, в человечески-ценном, в общественно значимом. Эта основная потребность объективации проявляется в стремлении расширить границы своего индивидуального бытия, воплотить, объективировать себя в формах, неподвластных течению времени, сделать свою жизнь более интенсивной в настоящем» (Абульханова-Славская, 1991: 72).

Только за последние 5-10 лет в таких ведущих мировых научных журналах, как The Journal of Neuroscience, Acta Psychologica, Developmental Psychology, Journal of Experimental Psychology, Human Perception \& Performance, Journal of Cognition \& Development, Child Neuropsychology, Child Psychology Clinical Neuropsychology, American Journal of Psychology, Journal of Cognitive Neuroscience и ар., было опубликовано свыше 100 исследований по тематике субъективного времени. 
По данным А. И. Мелехина (Мелехин, 2013: Электронный ресурс) динамику исследований можно представить в таблицах (табл. 1 и 2).

Таблииа 1

АИНАМИКА ИССАЕАОВАНИЙ ВРЕМЕНИ В ОТЕЧЕСТВЕННОЙ ПСИХОАОГИИ

В 1960-2010 гг., ПО ЧИСАУ ПУБАИКАЦИЙ

Table 1

TEMPORAL STUDIES BY DISCIPLINE WITHIN PSYCHOLOGY IN THE USSR AND RUSSIA, 1960-2010, BY NUMBER OF PUBLICATIONS

\begin{tabular}{|l|c|c|c|c|c|c|}
\hline & 1960 & 1970 & 1980 & 1990 & 2000 & 2010 \\
\cline { 2 - 7 } Общая психология & 2 & 6 & 7 & 12 & 26 & 57 \\
Педагогическая психология & 5 & 8 & 15 & 19 & 17 & 27 \\
Психофизиология & 7 & 11 & 17 & 21 & 34 & 78 \\
Возрастная психология & & & & & & 26 \\
\hline
\end{tabular}

Таблича 2

АИНАМИКА ИССАЕАОВАНИЙ ВРЕМЕНИ В ЗАРУБЕЖНОЙ ПСИХО ОГИИ

В 1960-2010 гг., ПО ЧИСАУ ПУБАИКАЦИЙ

Table 2

TEMPORAL STUDIES BY DISCIPLINE WITHIN PSYCHOLOGY OUTSIDE THE USSR AND RUSSIA, 1960-2010, BY NUMBER OF PUBLICATIONS

\begin{tabular}{|l|c|c|c|c|c|c|}
\hline & 1960 & 1970 & 1980 & 1990 & 2000 & 2010 \\
\cline { 2 - 7 } Общая психология & 5 & 8 & 10 & 17 & 19 & 89 \\
Педагогическая психология & 8 & 12 & 15 & 19 & 17 & 27 \\
Психофизиология & 11 & 18 & 24 & 34 & 59 & 112 \\
Возрастная психология & 12 & 18 & 23 & 43 & 67 & 93 \\
\hline
\end{tabular}

Из приведенных данных в таблицах очевидно, что современную психологию, как отечественную, так и зарубежную, больше интересует психофизиологическая сторона процесса восприятия времени в норме и патологии, нежели онтогенетическая сторона. По большей части исследования касаются роли различных психических функций в процессе восприятия времени: внимания, памяти и мышления, а также церебральных структур. Отечественная психология в изучении субъективного времени представлена именами С. А. Рубинштейна, К. А. Абульхановой, В. И. Ковалева, Б. И. Цуканова, Т. А. Нестика, В. И. Слободчикова и др.

Подробный обзор, охватывающий 125 лет изучения субъективного времени, дан П. Ханкоком (P. Hancock), P. Блоком (R. Block) в статье «Психология времени: взгляд назад и вперед» (Hancock, Block, 2012). Авторы отмечают новые направления изучения психологии времени в XXI в. и выражают надежду, что старые вопросы психологии времени об индивидуальных различиях получат новое развитие и что именно из 
развития темы индивидуальных различий вырастут новые продуктивные направления будущих исследований и теорий психологии времени. Новыми направлениями названы междисциплинарные исследования психологии времени. Они связывают эти направления в теме изучения психологии времени с научным обменом между отраслями знаний - биологией, медициной, нейронауками и психологией (см. также: Block, Hancock, Zakay, 2010).

Мы полагаем, что новизну в исследования психологического времени также привнесет естественнонаучный подход, основанный на объективных измерениях параметров течения психологического времени.

\section{ТЕМПОМЕТРИЯ}

Традиционный подход в психологии в русле «восприятия времени» рассматривает основным предметом изучения психологический феномен восприятия, а именно восприятия договорной условности, объективного феномена. В этом направлении изучаются именно восприятие времени и оценка временных интервалов - во всех областях психологии, в которых уже сделаны обширные и глубокие исследования, начиная со ставшими классикой исследований В. Вундта и продолжая сегодняшними многочисленными повторениями и приложениями в рамках восприятия времени, восприятия будущего, восприятия прошлого, взаимовлияния этих восприятий и т. п. Методика вербальных оценок восприятия времени, несмотря на свою продуктивность, остается опосредованной методикой исследования субъективного времени.

Сегодня такой подход, по нашему мнению, сужает область исследований. Мы считаем существенным ограничением общий подход к предмету как к субъективному времени вместо субъективных времен, а именно множества субъективных времен для одного человека. Такие теоретические посылки ограничивают горизонты для исследований и оставляют за полем исследования природу самого феномена - феномена психологического времени субъекта, т. е. субъективного времени. Mь предлагаем измерять, исследовать, анализировать в качестве предмета исследования само субьективное время. Тем самым, с точки зрения стороннего исследователя, психологическое время наблюдаемого человека выступает как объективная реальность, которую можно наблюдать и изучать. Таким образом, мы можем считать, что используем естественнонаучные методы исследования.

Аля нового подхода мы вводим в научный оборот новый термин для его обозначения - психологическая темпометрия. Продуцирование субъектом временных интервалов рассматривается как принципиально иной феномен по сравнению с феноменом восприятия объективного времени. Взаимосвязь и взаимовлияние процессов восприятия времени и межвременной выбор между субъективными временами также являются предметом исследований в психологической темпометрии (см.: К теории субъективных времен ... : Электронный ресурс).

Неологизм психологическая темпометрия предлагается использовать в нескольких значениях.

Психологическая темпометрия в самом широком значении этого слова означает ориентацию человека во временных отношениях с миром и на всех уровнях психического отражения (ощущений, эмоций, восприятий, представлений, уровни значений, понятийный уровень, уровень теорий, уровень формального мышления). Психологическая темпометрия имеет дело с содержанием таких ориентаций во времени, как чувство времени (pre-timing), дорациональное ориентирование, охватывая иррацио- 
нальные временные выборы (irrational grasp of time). Так, известное выражение «время - деньги» отражает ориентацию во временном отношении с миром на уровне ценностных значений. В физике время определяется на понятийном уровне отношений между человеком и миром, измеряя длительности в часах, минутах, сутках и др. На этом уровне оперирование понятием времени происходит иначе, чем на уровне ощущений и чувств, когда о времени говорят «быстро», «пролетело незаметно», «мучительно тянется» и др. Ориентация может происходить на разных уровнях психического отражения мира и себя в этом мире. В аксиоматической математике, на уровне формального мышления, время отражается как компонент пространственно-временных координат.

Психологическая темпометрия в более узком определении есть оперирование соотношениями временных векторов субъекта. Кроме этого, психологическая темпометрия есть динамическое соотношение человека с миром по каждому отдельному от других направлений субъективных времен, от начал отдельных субъективных времен до их окончаний (от возникновений до их завершений). При этом каждое отдельное время имеет свою динамическую картину возникновения, изменения и завершения.

Таким образом, психологическая темпометрия - это психическая активность человека особого рода по переживанию, ошушению и определению себя во временньх отношениях к миру. Сравнение движений и оценка темпа происходят на всех уровнях психического отражения мира и себя в мире. Это психическая активность ориентации в субъективных временах, активность оценивания течений деятельности на разных временных направлениях, сравнения временных смыслов, выборе субъективных направлений в субъективных временах, активность в сопоставлениях и выборе среди потребностей, квазипотребностей, целей, намерений, желаний. Это активность на основе своих ощущений, чувств, представлений, разворачивающаяся от ближайших миллисекундных и до отдаленных временных горизонтов космоса. Активность, названная Г. Зауерманом (G. Zauberman) и Б. Киу Кимом (B. Kуu Kim) как предвосхищаемое восприятие времени, - тоже из этого рода активности (Kim, Zauberman, 2009). Активность, для обозначения которой А. К. Нобр (А. С. Nobre) и его коллега Г. Роэнколь (G. Rohenkohl) предпочитают употреблять название «временное направление» (temporal orienting) (Nobre, Rohenkohl, 2009: Электронный ресурс), А. Буономано (D. Buonomano) и У. Кармакар (U. Karmakar) называют это временным процессингом, межмодальным таймингом (temporal processing, intermodal timing) (Karmakar, Buonomano, 2007).

\section{ТЕОРЕТИЧЕСКИЕ ИСТОЧНИКИ ПСИХОАОГИЧЕСКОЙ ТЕМПОМЕТРИИ}

В становлении психологической темпометрии следует особо отметить значение работы С. $\Lambda$. Рубинштейна «Восприятие времени», являющейся не только главой лучшего учебника психологии «Основы общей психологии», но и глубоким теоретическим анализом проблемы психологического времени (Рубинштейн, 2002: 249-256). Несмотря на то что С. $\Lambda$. Рубинштейн рассматривает феномен восприятия времени, именно этот труд содержит теоретические положения, которые предвосхищают развитие нового направления психологии - психологической темпометрии.

Книга С. А. Рубинштейна «Человек и мир» содержит предположение об «объективности» субъективного времени, его относительности для наблюдателя и наблюда- 
емого: «Субъективно переживаемое время - это не столько кажущееся, в переживании якобы неадекватно преломленное время движущейся материи, а относительное время жизни (поведения) данной системы - человека, вполне объективно отражающее план жизни данного человека. В концепции времени отражается теория детерминации процесса» (Рубинштейн, 2012: 47).

В основе развития психологической темпометрии лежат основные положения и предположения С. $\Lambda$. Рубинштейна по теме восприятия времени, там же мы ищем перспективные линии для научного поиска. Обращаясь к работам С. . Рубинштейна о восприятии времени как к теоретическому первоисточнику, можно найти основания и направления для продуктивного развития исследований субъективного времени и переходу от «чувства времени» к непосредственно феномену субъективного времени.

В работе «Восприятие времени» С. А. Рубинштейна содержится важное положение, что полное разрешение трудностей, связанных с текучей диалектикой временных определений, требует принятия одной общей системы координат с постоянной точкой. Аля разрешения этих трудностей предлагается эту постоянную точку рассматривать как движущуюся в объективном времени и, кроме этого, рассматривать все вектора времен («вектор времени»- термин С. $\Lambda$. Рубинштейна) исходящими из этой движущейся точки. Начала всех векторов возникают в этой движущейся точке времени. Вектора направлены на предметы потребностей, квазипотребностей, желаний субъекта. Вектора в предметном плане устремлены по разным направлениям на расположенные в предметном поле предметы потребностей. Во временном плане, в самом первом приближенном определении, вектора направлены из этой точки в будущее. Вектора в движущейся точке на объективном времени, в которой находится одна общая система координат, меняются как по мере течения объективного времени, так и по волеизъявлению субъекта. Эта точка - точка настоящего мгновения жизни. Все эти вектора субъективных времен вместе с тем параллельны друг другу и параллельны ходу объективного времени. Имеет место движущаяся параллельность векторов из настоящего в будущее. Вектора различаются так, что через «призму» этих векторов восприятие одного и того же интервала объективного времени по разным направлениям различно. Если использовать геометрическую, т. е. пространственную, аналогию в темпометрии, то это выглядит как различные проекции разных времен на общую шкалу объективного времени. Восприятие времени (по сути — проецирование какого-либо интервала объективного времени на разные вектора субъективных времен) в этой аналогии будет проекцией воспринимаемой длительности на разные субъективные шкалы. Изменения шкалы возможны как для сжатия, так и для растяжения. Такое помещение векторов на движущейся точке является новым подходом к описанию субъективно-временной динамики.

Все субъективное время представляется как пучок субъективных времен, параллельных объективному времени. Субъективное время - многомерное «пространство» векторов. Субъективное время - набор направленных (делений, биений, колебаний) времен. Такое представление субъективных времен как системы координат, в которой происходит ориентировка во времени, соответствует определению времени С. $\Lambda$. Рубинштейном как вектора: «поскольку время - направленная величина (вектор)» (Рубинштейн, 2002: 304).

Кроме направленных величин - векторов, имеют место возникающие напряжения. «При незаполнении же переживаемого времени в переживании обычно создает- 
ся томительное напряжение», искажающее восприятие времени (там же: 303). Напряженное направление по времени может быть напряжено двояко: имеет место «натянутое» или, наоборот, «растянутое время». «Время ожидания желательного события в непосредственном переживании томительно удлиняется, нежелательного - мучительно сокращается», - пишет С. $\Lambda$. Рубинштейн (там же). Эти два по-разному искажающих время варианта представляют по-разному напряженный вектор. Математическим аналогом можно считать тензор (расширение понятия «вектор»на пространства векторов и функционалы над векторным пространством). Основная сложность, возникающая на пути применения тензорного исчисления, состоит в том, что сами координаты меняются. И эти координаты суть вектора из настоящего мгновения в будущее. Пока не было никаких попыток применить тензорное исчисление к такой подвижной системе координат, привязанной к настоящему мгновению, системе, в которой время может удлиняться и укорачиваться.

Отмеченная С. А. Рубинштейном господствующая (доминирующая) направленность, как она есть в диалектическом движении, подразумевает выбор и последующую господствующую направленность. Свободный человек обречен делать бессознательный, интуитивный или сознательный выбор также и среди временных направлений. Уже отсюда следует, что субъективное время не одно, а несколько и имеет разные направления. Такое естественное расширение следует из отмеченной С. $\Lambda$. Рубинштейном направленности, имеющей свойство детерминанты. Результирующая, определяющая направленность субъективного времени возникает на сделанном сравнении и на выборе по меньшей мере из двух направленностей и признании, осознании и выборе, в какой-либо форме ощущения или сознательного выбора личностью этой господствующей направленности. Поскольку речь идет о переживаемой длительности и ее отклонениях от объективного времени в ту или иную сторону, то возникает проблема: как именно среди нескольких направленностей (векторов, тензоров) происходит выбор, каковы как на бессознательном, так и на сознательном уровне психического отражения механизмы выбора субъективного времени у личности.

Остаются вопросы и о том, на каком уровне психического отражения такой выбор происходит. Эти вопросы можно сформулировать иначе: продолжать ли вслед за В. Вундтом и С. $\Lambda$. Рубинштейном обрамлять в кавычки «чувства времени» и «чувство времени». Приведем в качестве примера слова С. $\Lambda$. Рубинштейна: «У нас имеется некоторое непосредственное переживание, ощущение или “чувство” времени» (Рубинштейн, 2002: 302). Аиалектический подход С. А. Рубинштейна обязывает рассматривать противоположности явления в их развитии.

Рассмотрим текучую диалектику временных определений на конкретном примере. Как переживаемая длительность отклоняется в случае двух разных направленностей у водителя автотранспорта в ситуации обгона попутного авто по встречной полосе движения: одна - быстрее двигаться к пункту назначения и вторая - не попасть в АТП, остаться в живых? Желательное событие - успешно обогнать попутный автомобиль при отсутствии машин на встречной полосе. Нежелательное событие столкновение со встречным автомобилем. Рассмотрим прежде по отдельности каждую из этих направленностей, допуская в разных случаях наличие одной и отсутствие другой. В одном случае перед нами ситуация обгона, когда нет встречных машин, в другом - когда идет поток встречных машин и даже не возникает желания обгонять. Когда же наш водитель идет на обгон, то субъективное время завершения обгона действительно удлиняется. Во втором случае есть вероятность, что кто-то из 
встречного потока может оказаться на пути нашего водителя. У нашего водителя субъективное время ожидания того, когда приблизится встречная машина, также изменяется.

Но все здесь не так однозначно, как кажется. Многое зависит от позиции наблюдателя, а конкретнее - от содержательного наполнения направленностей. Ава вектора субъективных времен рисуют картину восприятия времени, и это двоякое восприятие времени. Единство и борьба противоположных восприятий разных субъективных времен, смена одного восприятия другим, сопоставление и снятие временных противоречий, диалектические переходы в качество - все это составляет в рассматриваемом примере содержание человеческой темпометрии. Аействительно, водителю кажется, что с, одной стороны, время тянется медленно и было бы хорошо успеть. С другой стороны, время течет быстро, и, воспринимая движение встречного автомобиля, водитель оценивает время: возможно или невозможно успеть. Вариации в разных желательных/нежелательных системах отсчета разные, зависящие от того, как ситуацию определяет сам водитель. «Успеть» и «не успеть», «пора», «рано», «позАно», «скоро»- временные характеристики. «Успеваю» или «не успеваю»- в таких ощущениях, «чувствах», представлениях оформляется результат происходящих оценок и влияния господствующей направленности. Имеет место «забегание» по времени в будущее. Имеет место антиципация в двух временах и выбор: обгонять или нет в соответствии с господствующей направленностью. (Нормальная, трезвая господствующая направленность - инстинкт самосохранения.)

\section{ЗАКАЮЧЕНИЕ}

Итак, традиционный подход в психологии в русле «восприятия времени», рассматривающий основным предметом изучения психологический феномен восприятия, по нашему мнению, сужает область исследований. Мы предлагаем измерять, исследовать, анализировать в качестве предмета исследования само субъективное время и сформулировали несколько предварительных гипотез новой концепции - концепции психологической темпометрии: 1) любая сильная потребность меняет течение субъективного времени, направленного на предмет потребности; 2) активность человека в каждый момент физического времени определяется самым актуальным субъективным временем в системе субъективных времен, направленным на самую актуальную (имеющую самую высокую величину напряженности) в данный промежуток физического времени потребность (квазипотребность, желание) индивида; 3) напряженность субъективного времени в системе субъективных времен взаимосвязана со степенью когнитивной и эмоциональной нагрузок человека; 4) в каждый момент времени человек переживает доминирующую потребность, и одно из субъективных времен, направленное на доминирующую потребность личности, имеет наибольшее напряжение в каждый момент.

Мы опираемся на сформулированный С. $\Lambda$. Рубинштейном закон заполненного временного отрезка (интервала). Следствие из этого закона - пустых, бессодержательных временных отрезков не существует. Мы применяем понятие вектора к субъективным временам личности и вводим дополнительную характеристику вектора «напряжения» направленного временного отрезка. Также мы предлагаем решение проблемы «текучей диалектики временных определений», предлагая координатную систему единиц измерений субъективного времени, привязанную к естественной отправной точке, коей является настоящее мгновение. 


\section{СПИСОК АИТЕРАТУРЫ}

Абульханова-Славская, К. А. (1991) Стратегия жизни. М. : Мысль. 299 с.

Зинченко, В. П. (2001) Время - действующее лицо // Вопросы психологии. № 6. С. 36-54.

К теории субъективных времен. Выведение метода из теории [Электронный ресурс] // $\Lambda$ боратория темпометрии. URL: http://tempometry.ru/cntnt/rus/left-column-rus/teoriya-subektivnih-vremen-vived.html [архивировано в WebCite] (дата обращения: 10.12.2014).

Мелехин, А. И. (2013) Исследования времени в отечественной психологии: наследие и перспективы [Электронный ресурс] // Институт исследований природы времени. 3 февраля. URL: http://chronos.msu.ru/old/RREPORTS/melehin_issledovaniya_vremeni.html [архивировано в WebCite] (дата обращения: 10.12.2014).

Рубинштейн, С. А. (2002) Основы общей психологии. СПб. : Питер. 720 с.

Рубинштейн, С. А. (2012) Человек и мир. СПб. : Питер. 224 с.

Block, R. A., Hancock, P. A., Zakay, D. (2010) How cognitive load affects duration judgments: A meta-analytic review // Acta Psychologica. Vol. 134. Issue 3. P. 330-343. DOI: 10.1016/j.actpsy.2010.03.006

Hancock, P. A., Block, R. A. (2012) The psychology of time: A view backward and forward // The American Journal of Psychology. Vol. 125. No. 3. P. 267-274. DOI: 10.5406/amerjpsyc.125.3.0267

Karmakar, U. R., Buonomano, D. V. (2007) Timing in the absence of clocks: Encoding time in neural network states // Neuron. Vol. 53. Issue 3. P. 427-438. DOI: 10.1016/j.neuron.2007.01.006

Kim, B. K., Zauberman, G. (2009) Perception of anticipatory time in temporal discounting // Journal of Neuroscience, Psychology, and Economics. Vol. 2. No. 2. P. 91-101. DOI: 10.1037/a0017686

Nobre, A. C., Rohenkohl, G. (2014) Time for the fourth dimension in attention // The Oxford handbook of attention / ed. by A. C. Nobre, S. Kastner. Oxford ; N. Y. : Oxford University Press. 1242 p. P. 676-721.

Дата поступления: 15.06.2015 2.

\section{THE RISE OF TEMPOMETRY AS A NEW THEORY \\ IN PSYCHOLOGY \\ S. V. TIKHOMIROVA \\ (INSTITUte of PSyChOlogy, RUSSIAN ACADEMY OF SCIENCES)}

Psychology has been dealing with the problem of subjective time for quite a while by now. In the last 50 years, there was a striking growth in the number of psychological studies of subjective time. Contemporary psychology, in Russia as well as abroad, takes more interest in psycho-physiological aspects of normal and pathological time perception rather than in the ontological and developmental aspects of the problem. Current studies focus on the role of various psychological functions of time perception - attention, memory and thinking, as well as on cerebral structures. In this article, we introduce tempometry as a new approach in psychology. Never before in Russia has the study of subjective time been founded on the approach developed by natural science.

A shift from studying time perception to the phenomenon of psychological time proper is a seachange in itself. Interconnection and mutual influence of time perception and a choice between subjective times are also the objects of psychological telemetry. Theoretical foundations of the new approach can be found in the works of S. L. Rubinshtein. Although he remained within the paradigm of time perception studies, we find it impossible to overestimate the importance of theoretical ideas found in the chapter on "Time Perception" in the best psychology textbook - "Foundations of General Psychology" - that he wrote. In the book, he also provides a profound theoretical analysis of psychological time. Among the most important ideas originally put forward by S. L. Rubinstein and to be further developed in psychological tempometry, are the concept of vector applied to subjective time; the problem of "fluent dialectic of time definitions"; the concept of tension within a segment of time; the law of filled-in time intervals; a system of coordinates for the units of subjective time measurement, with the current moment acting as its natural pivot; and future as priority focus in the currently experienced. 
Keywords: tempometry; subjective time; natural science approach in psychology; S. L. Rubinstein; time perception

\section{REFERENCES}

Abulkhanova-Slavskaia, K. A. (1991) Strategiia zhizni [The strategy of living]. Moscow, Mysl' Publ. 299 p. (In Russ.).

Zinchenko, V. P. (2001) Vremia - deistvuiushchee litso [Time as a subject]. Voprosy psikbologii, no. 6, pp. 36-54. (In Russ.).

$\mathrm{K}$ teorii sub'ektivnykh vremen. Vyvedenie metoda iz teorii [Towards the theory of subjective time. Deducing the method from the theory]. Laboratoriia tempometrii [Tempometry Lab] [online] Available at: http://tempometry.ru/cntnt/rus/left-column-rus/teoriya-subektivnih-vremen-vived. html [archived in WebCite] (accessed 10.12.2014). (In Russ.).

Melekhin, A. I. (2013) Issledovaniia vremeni v otechestvennoi psikhologii: nasledie i perspektivy [Temporal studies in Russian psychology: Legacy and prospects]. Institut issledovanii prirody vremeni [Institute for Time Nature Explorations]. February 3. [online] Available at: http://chronos. msu.ru/ old/RREPORTS/melehin_issledovaniya_vremeni.html [archived in WebCite] (accessed 10.12.2014). (In Russ.).

Rubinshtein, S. L. (2002) Osnovy obshchei psikbologii [Foundations of general psychology]. St. Petersburg, Piter Publ. 720 p. (In Russ.).

Rubinshtein, S. L. (2012) Chelovek $i$ mir [Man and the world]. St. Petersburg, Piter Publ. 224 p. (In Russ.).

Block, R. A., Hancock, P. A. and Zakay, D. (2010) How cognitive load affects duration judgments: A meta-analytic review. Acta Psychologica, vol. 134, issue 3, pp. 330-343. DOI: 10.1016/j.actpsy. 2010.03.006

Hancock, P. A. and Block, R. A. (2012) The psychology of time: A view backward and forward. The American Journal of Psychology, vol. 125, no. 3, pp. 267-274. DOI: 10.5406/amerjpsyc.125.3.0267

Karmakar, U. R. and Buonomano, D. V. (2007) Timing in the absence of clocks: Encoding time in neural network states. Neuron, vol. 53, issue 3, pp. 427-438. DOI: 10.1016/j.neuron.2007.01.006

Kim, B. K. and Zauberman, G. (2009) Perception of anticipatory time in temporal discounting. Journal of Neuroscience, Psychology, and Economics, vol. 2, no. 2, pp. 91-101. DOI: 10.1037/ a 0017686

Nobre, A. C. and Rohenkohl, G. (2014) Time for the fourth dimension in attention. In: The Oxford bandbook of attention / ed. by A. C. Nobre and S. Kastner. Oxford ; New York, Oxford University Press. 1242 p. Pp. 676-721.

Submission date: 15.06.2015.

Тихомирова Светлана Витальевна - кандидат психологических наук, научный сотрудник лаборатории социальной и экономической психологии Института психологии Российской академии наук. Адрес: 129366, Россия, г. Москва, ул. Ярославская, д. 13, корп. 1. Тел.: +7 (495) 682-42-07. Эл. aApec: sti@ipras.ru

Tikhomirova Svetlana Vitalievna, Candidate of Psychology, Research Fellow, Laboratory of Social and Economic Psychology, Institute of Psychology, Russian Academy of Sciences. Postal address: Bldg. 1, 13 Yaroslavskaya St., 129366 Moscow, Russian Federation. Tel.: +7 (495) 682-42-07. E-mail: sti@ipras.ru 